\title{
LAPURDUTI
}

Euskal ikerketen aldizkaria | Revue d'études basques |

Revista de estudios vascos | Basque studies review

$8 \mid 2003$

Numéro VIII

\section{L'évolution diachronique de l'expression de la possibilité en basque}

\section{Gerd Jendraschek}

\section{OpenEdition \\ Journals}

Édition électronique

URL : http://journals.openedition.org/lapurdum/1143

DOI : 10.4000/lapurdum. 1143

ISSN : 1965-0655

Éditeur

IKER

Édition imprimée

Date de publication : 1 novembre 2003

Pagination : 263-283

ISBN : 9782867813436

ISSN : $1273-3830$

Référence électronique

Gerd Jendraschek, «L'évolution diachronique de l'expression de la possibilité en basque », Lapurdum [En ligne], 8 | 2003, mis en ligne le 01 mai 2009, consulté le 20 avril 2019. URL : http:// journals.openedition.org/lapurdum/1143; DOI : 10.4000/lapurdum.1143 


\section{Gerd Jendraschek}

Equipe de Recherche en Syntaxe et Sémantique (ERSS),

Université de Toulouse-Le Mirail

\section{L'évolution diachronique de l'expression de la possibilité en basque}

\section{Résumé}

En basque, l'expression des notions modales de possibilité, de capacité et de l'épistémique a considérablement évolué depuis le 16e siècle. Le nombre des différentes formes d'un potentiel synthétique construit à l'aide du suffixe modal -ke s'est réduit. Certaines formes ont été fossilisées et ont adopté un sémantisme plus restreint. En basque classique, des particules étaient nécessaires pour désambiguer les diverses fonctions des auxiliaires au mode du potentiel. En basque moderne, on emploie soit la particule, soit l'auxiliaire complexe, mais rarement les deux ensemble. Des formes qui servaient à l'expression d'un futur indéterminé sont devenues des marqueurs épistémiques, mais ces formes sont également en train de disparaître. Par contre, il reste difficile d'employer la particule modale ahal dans un contexte épistémique. Alors que les constructions avec l'auxiliaire et celles avec la particule sont équivalentes lorsque le contexte n'est pas épistémique, des formes construites avec le suffixe modal - $k e$ sont préférées lorsqu'il ne s'agit pas d'une capacité, mais d'une hypothèse. Finalement, on va voir que les variétés du nord et celles du sud se distinguent par la manière d'exprimer la possibilité et sa négation. Une même forme al/ahal était employée comme particule interrogative au sud, mais comme particule de possibilité au nord, ce qui peut être un élément d'explication. Avec l'émergence de variétés supradialectales, les deux interprétations commencent à se côtoyer sous des graphies distinctes. En résumé, les différents exemples montrent que deux variétés d'une même langue somme toute relativement proches peuvent exhiber des différences grammaticales assez prononcées.

\section{L'évolution des particules modales ahal et ezin}

La conjugaison basque est largement périphrastique. Le complexe verbal est composé d'un participe qui indique l'aspect, et d'un auxiliaire indiquant le temps, le mode et les personnes. Il existe une série de particules qui 
se placent entre le participe et l'auxiliaire et qui expriment des catégories modales ou aspectuelles. Comme elles contiennent des informations qui complètent celles données par l'auxiliaire, on peut les appeler "particules auxiliaires". Parmi ces particules, on regardera par la suite les comportements divergents de ahal qui exprime une possibilité ou une capacité, et ezin qui exprime une impossibilité ou une incapacité.

\subsection{La situation en basque classique}

Les deux particules se trouvent déjà dans les textes les plus anciens qui datent du $16^{\mathrm{e}}$ et $17^{\mathrm{e}}$ siècle. E1 montre que ahal s'y combine avec une forme de l'auxiliaire, à savoir dezakezu, qui contient elle-même une marque de modalité potentielle, le suffixe $-k e$ (voir section 2.1.). L'orthographe des exemples a été adaptée aux conventions actuelles.

$\begin{array}{ccccc}\text { E1. Paga } & \text { ahal } & \text { dezakezu-la, } & \text { paga-tu } & \text { gabe, } \\ \text { BAS payer } & \text { pouvoir } & \text { PRS.SUBJ.POT-SR } & \text { payer-PART } & \text { sans } \\ & & \text { 3.SG<2.SG } & & \end{array}$

jabe-a-ren borondate-a-ren kontra, on-a-ren eduki-tze-a, proprétaire-DET-GEN volonté-DET-GEN contre bier-DET-GEN tenir-NR-DET bekatu da.

péché PRS.3.SG

"Quand tu peux payer, c'est un péché de posséder un bien sans payer et contre la volonté du propriétaire."

(Axular 1643, Gero)

La modalité est alors exprimée deux fois, et l'on pourrait analyser cette co-occurrence comme une sorte de dépendance syntagmatique entre la particule et le suffixe $-k e$. Ce dernier ne pouvait alors pas exprimer la possibilité indépendamment, probablement parce que sa fonction était plus floue qu'elle ne l'est aujourd'hui. Ce "suffixe d'indétermination" était employé pour indiquer que l'information était moins précise, moins certaine et moins pressante, qu'elle n'était pas réelle, mais seulement une éventualité, un événement virtuel. Il pouvait s'agir d'une action future, possible ou "intemporelle" (Lafon 1970/1999 : 492-494). Après les particules ahal ou ezin, la forme dezakezu était alors préférée à la forme non marquée de l'auxiliaire, duzu, 
parce qu'elle êtait, par son caractère "virtuel", plus compatible avec le sémantisme des particules. Inversement, les particules permettaient de désambiguer les diverses fonctions des formes en -ke mentionnées ci-dessus.

E2 montre que ezin, à la différence des autres particules, se place devant la forme du verbe lexical, ce qui s'explique par sa fonction de négation.

$\begin{array}{lllll}\text { E2. } & \text { Ba-dirudi } & \text { ez } & \text { de-la honela-ko } & \text { obra-rik: } \\ \text { BAS } & \text { AFF-sembler:PRS.3.SG } & \text { NEG } & \text { PRS.3.SG-SR tel-MR } & \text { oeuvre-PRTF }\end{array}$

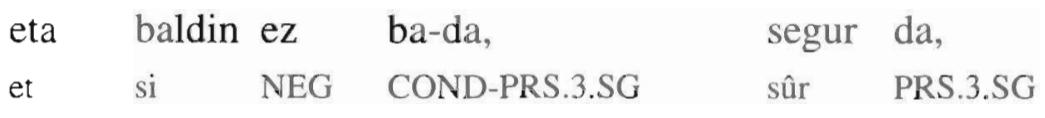

$\begin{array}{llllllll}\text { ezin } & \text { paga } & \text { dezakegu-la } & \text { geure } & \text { Jainko-a, eta } & \text { ez } & \text { satisfa. } \\ \text { NPOT } & \text { payer } & \text { PRS.SUBJ.POT-SR } & \text { POSS.1.PL } & \text { Dieu-DET } & \text { et } & \text { NEG } & \text { satisfaire } \\ & & 3 . S G<1 . P L & & & & & \end{array}$

"Il semble qu'il n'y a pas de telle œuvre : et s'il n'y en a pas, c'est sûr, c'est que nous ne pouvons pas payer notre Dieu, ni le satisfaire."

(Axular 1643, Gero)

Mis à part l'ordre des éléments, les deux constructions sont similaires. Elles sont composées d'une particule modale, ahal à l'affirmative, ezin dans la négation, du radical du verbe lexical (dépourvu de marques aspectuelles), et de la forme potentielle du subjonctif de l'auxiliaire. On peut supposer que cette construction était la plus répandue en basque ancien (du nord au moins), car on trouve chez l'écrivain Leizarraga des constructions comme ikus ahal daitezke "ils peuvent être vus" et barka ahal ditzake "il peut les pardonner".

\subsection{L'évolution en basque du nord}

Au fil du temps, ces constructions ont évolué et on commence à les considérer comme étant des pléonasmes (Lafitte 1944 : 350). La double expression de la modalité est alors abandonnée, car la possibilité est désormais exprimée soit par la particule seule, soit par la forme modale de l'auxiliaire, sans la particule. Comme E1 et E2 proviennent de sources du Pays basque nord, on va d'abord regarder comment ces constructions ont évolué dans les dialectes du nord. Avant de continuer, précisons que les varié- 
tés de Haute-Navarre ne seront pas prises en compte ici parce qu'elles constituent des variétés de transition entre celles du nord-est (Labourd, BasseNavarre, Soule) et celles du sud-ouest (Guipuscoa, Biscaye). Quand il est question des variétés méridionales, il est donc important de savoir que la description ne s'applique pas nécessairement de la même manière au haut-navarrais.

Dans toutes les variétés du basque, on peut constater une tendance à éviter des formes synthétiques qui sont perçues comme morphologiquement trop complexes. Ce processus de simplification morphologique est plus avancé en basque du nord que dans les variétés du sud. Ainsi, les formes de possibilité de l'auxiliaire comme dezakezu (cf. E1 et section 2.1.) sont aujourd'hui rares dans le nord, alors qu'elles restent très répandues dans le sud. Comme ces formes sont abandonnées et remplacées par les formes non marquées correspondantes ( $d u z u$ au lieu de dezakezu, da au lieu de daiteke), la particule ahal seule exprime la modalité. E3 est un exemple contemporain.

$\begin{array}{llllll}\text { E3. } & \text { Memento-an eros-te-n } & \text { ahal da } & \text { amo-a, artain-a, } & \text { etxe } & \text { ogi-a. } \\ \text { BAS moment-LOC acheter-NR-LOC } & \text { pouvoirPRS.3.SG } & \text { vin-DET poisson-DET maison } & \text { pain-DET }\end{array}$

"Pour l'instant, on peut acheter du vin, du poisson et du pain de maison."

(www.herriak.info/lapurdi)

Au lieu du radical, les variétés orientales du basque (celles du nord plus le haut-navarrais) combinent ahal avec le participe de l'imperfectif en t(z)en qui est composé étymologiquement d'un infinitif nominalisé en -t(z)e et du locatif en $-n$. Au lieu de la construction paga ahal dezakezu qu'on a vue en $\mathrm{E} 1$, on dirait alors pagatzen ahal duzu.

Pour exprimer l'impossibilité, le basque classique employait la construction ezin + radical + dezake/daiteke. Cette construction est rare aujourd'hui. Au nord, la négation d'une construction de possibilité avec ahal se fait comme la négation de n'importe quelle autre proposition, à savoir à l'aide de la particule de négation ez. Pour exprimer l'impossibilité, ez se combine alors avec ahal (E4). 


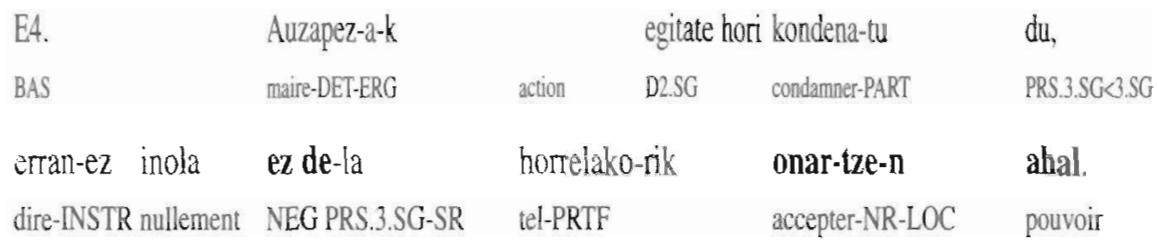

“Le maire a condamné cette action en disant qu'on ne pouvait nullement accepter de telles choses."

(www.herriak.info/lapurdi)

Au lieu de la construction ezin paga dezakegu vue en E2 on obtiendrait par conséquent ez dugu pagatzen ahal.

\subsection{L'évolution en basque du sud}

Au sud, l'évolution a été complètement différente. Regardons d'abord les constructions affirmatives. Ici, ce n'est pas la particule qui exprime la possibilité, mais la forme marquée de l'auxiliaire (dezakeldaiteke etc.). En général, celle-ci se combine avec le radical, mais le participe du perfectif n'est pas rare dans cette position (King 1994:381;260). On trouve alors paga dezakezu ou pagatu dezakezu pour "tu peux payer". L'emploi du radical paga dans cette construction correspond à un usage littéraire, tandis que la forme pagatu est répandue dans le style familier. Cette variation stylistique peut être considérée comme étant la conséquence du processus de standardisation qui a réintroduit la distinction fonctionnelle entre le radical et le participe du perfectif. Traditionnellement, les deux formes étaient en variation libre ou dialectale dans le sud (King, comm. pers.).

Dans les variétés du sud-ouest, la particule ahal n'est employée que lorsque une forme correspondante de l'auxiliaire n'est pas disponible, c'està-dire dans certains temps composés (futur, passé récent, passé lointain), dans certaines propositions subordonnées, ou dans certaines locutions comme par exemple ahal duen guztia "tout ce qu'il/elle peut".

Dans les propositions négatives, c'est l'inverse qui s'est produit. A la différence du basque du nord, la particule ezin y a mieux résisté pour exprimer l'impossibilité, mais comme au nord, c'est l'auxiliaire simple qui est le plus souvent employé (E5). 


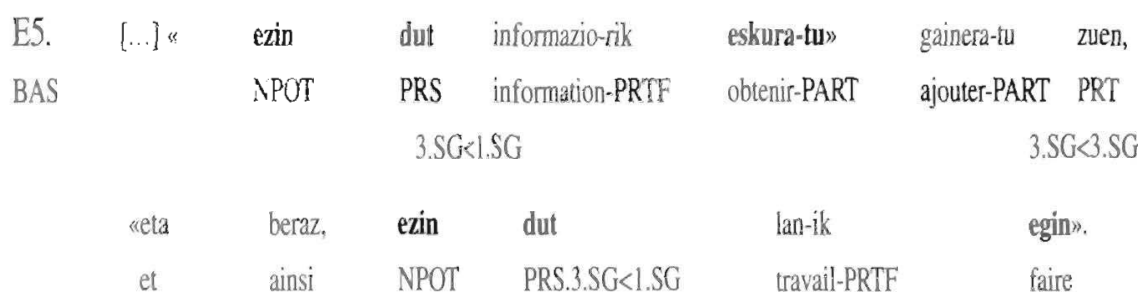

"“`Je ne peux pas obtenir d"information" a-t-il ajouté, "et ainsi, je ne peux pas travailler","

(www.egunero.info)

\subsection{Ambiguïtés sémantiques de la particule ahal}

Avant de continuer, résumons les différentes constructions. Les textes anciens montrent que les formes qu'emploient les variétés modernes dans le domaine de la possibilité ne sont pas des innovations, mais plutôt des constructions simplifiées, avec des préférences différentes. T1 montre les différentes constructions traduisant "tu (ne) peux (pas) payer".

\section{T1. Les différentes constructions de possibilité}

\begin{tabular}{|ccc|}
\hline variété & affirmatif & négatif \\
\hline classique & paga ahal dezakezu & ezin paga dezakezu \\
\hline nord & pagatzen ahal duzu & ez duzu pagatzen ahal \\
\hline sud & paga(tu) dezakezu & ezin duzu pagatu \\
\hline
\end{tabular}

Comment se fait-il que ahal soit moins employé au sud qu'au nord ? Il n'est pas sûr que le modèle classique des auteurs navarro-labourdins (les principaux dialectes du nord) soit aussi celui du guipuscoan de cette époque. Ce qui est pourtant sûr, c'est que le lexème ahal existait partout car il est la base d'une série de dérivés comme a(ha)lmen "capacité" ou a(ha)legin "effort", qui sont déjà attestés dans des sources du début du vingtième siècle. On constate pourtant que son évolution comme marqueur grammatical n'est pas la même selon les régions. Il est sans doute important de noter que la graphie moderne ahal représente la prononciation du nord où l'aspiration (représentée par le graphème $<\mathrm{h}>$ ) s'est mieux conservée. Au Guipuscoa, on prononçait plutôt $a l$, et on employait cette forme pour transformer une proposition en question. L'emploi d'un auxiliaire pour exprimer la possibilité évitait de possibles confusions avec l'autre fonction de $a(h a) l$. Dans les variétés du 
nord, la fonction interrogative de la particule était marginale (cf. Zubiri \& Zubiri 2000 : 578). Lafitte note que "la particule ahal placée entre la négation ez ou encore l'affirmation ba et la forme personnelle du verbe donne à la phrase une nuance d'interrogation rassurée à sens optatif' et il donne l'exemple reproduit ici comme E6 (Lafitte 1944/2001 : 99). Dans ce sens, la particule se combine aussi avec des formes verbales au futur (E7, comm. pers. de Xarles Videgain).

$\begin{array}{lllll}\text { E6. } & \text { Ez } & \text { ahal } & \text { nute } & \text { ikus-i? } \\ \text { BAS } & \text { NEG } & \text { INT } & \text { PRS.1.SG<3.PL } & \text { voir-PART }\end{array}$

"Ils ne m'ont pas vu, j'espère?"

$\begin{array}{lllll}\text { E7. } & \text { Ez } & \text { ahal } & \text { da } & \text { jin-en. } \\ \text { BAS } & \text { NEG } & \text { INT } & \text { PRS.3.SG } & \text { venir-FUT } \\ & \text { “(J'espère qu') Il ne viendra pas." }\end{array}$

Pour marquer une question neutre, les variétés du nord, notamment le bas-navarrais et le souletin, préfèrent le suffixe $-a$ (dızu devient par exemple duzuia). On peut encore mentionner une autre particule, à savoir ote : celleci n'a pas exactement le même sémantisme que ahal, car la plupart des locuteurs, surtout au sud, l'associent avec l'expression d'un doute ("Je me demande...").

Autrefois, l'emploi interrogatif de $a(h a) l$ était limité en dehors du guipuscoan, mais il s'est répandu avec la standardisation, car il est aujourd' hui très fréquent dans le langage journalistique. Avant, une phrase comme E8 pouvait être interprétée de manière différente selon les régions (si l'on ignore les différences prosodiques).
E8. Liburu-a uler-tze-n
a(ha)l
duzu.
BAS livre-DET comprendre-NR-LOC pouvoir/INT
PRS.3.SG $<2 . S G$
1. "Tu peux comprendre le livre."
(labourdin)
2. "Est-ce que tu comprends le livre ?" (guipuscoan) 
En basque moderne, la graphie distingue les deux emplois. Ainsi, pour marquer une question, on adopte la prononciation du guipuscoan et on écrit $a l$, tandis que la particule de possibilité est maintenant partout écrite selon la prononciation du nord, à savoir ahal. La polysémie a donc été supprimée par le recours a différentes prononciations régionales. Cette polysémie peut expliquer l'assymétrie entre la particule affirmative ahal et son équivalent négatif ezin. En effet, là où ahal est employé en basque méridional, la polysémie est rêsolue par la syntaxe. On pourrait en déduire que ahal n'a été maintenu que dans les cas où la syntaxe permettait de désambiguer les deux interprétations, comme c'est le cas du passé récent (E9 vs. E10) ou du futur (E11 vs. E12).

$\begin{array}{llllll}\text { E9. } & \text { Liburu-a } & \text { eros-i } & \text { a(ha)l } & \text { izan } & \text { duzu. } \\ \text { BAS } & \text { livre-DET } & \text { acheter-PART } & \text { pouvoir } & \text { AUX.INTR } & \text { PRS.3.SG<2.SG }\end{array}$

"Tu as pu acheter le livre"

E10. Liburu-a eros-i al duzu?

BAS livre-DET acheter-PART INT PRS.3.SG<2.SG

"Est-ce que tu as acheté le livre?"

E11. Liburu-a eros-i a(ha)l izan-go duzu.

BAS livre-DET acheter-PART pouvoir AUX.INTR-FUT S.3.SG<2.SG

"Tu vas pouvoir acheter le livre"

$\begin{array}{lllll}\text { E12. } & \text { Liburu-a } & \text { eros-i-ko } & \text { al } & \text { duzu ? } \\ \text { BAS } & \text { livre-DET } & \text { acheter-PART-FUT } & \text { INT } & \text { PRS.3.SG<2.SG }\end{array}$

"Est-ce que tu vas acheter le livre?"

T2 résume la répartition des différentes constructions. Le basque septentrional et méridional est représenté par leurs dialectes dominants respectifs. Pour ce qui est du label 'standard' utilisé ici, il désigne les formes les plus courantes à l'écrit, toutes variétés régionales confondues. Il s'agit par conséquent de formes de prestige qui, même si elles sont localement peu employées à l'oral, sont mieux représentées dans les registres soutenus. 
T2. Les différentes interprétations de a(ha)l

\begin{tabular}{|c|c|c|c|}
\hline variété & potentiel présent & potentiel passé récent & interrogatif \\
\hline navarro-labourdin & 1 pagatzen ahal duzu & pagatu ahal izan duzu & $\begin{array}{l}\text { pagatzen duzuial } \\
\text { pagatzen ote duzu }\end{array}$ \\
\hline guipuscoan & paga(tu) dezakezu & pagatu al izan dezu & pagatzen al dezu \\
\hline standard & paga dezakezu & pagatu ahal izan duzu & pagatzen al duzu \\
\hline
\end{tabular}

\section{La conjugaison potentielle}

\subsection{La morphologie de la conjugaison potentielle}

Les formes du type dezake et daiteke contiennent un suffixe - ke qui leur confère une valeur virtuelle. Il s'agit d'un suffixe modalisant que l'on retrouve dans un certain nombre de formes exprimant des hypothèses, des suppositions, des possibilités et le futur. Les auxiliaires du type dezake (transitif) et daiteke (intransitif) sont construites sur la base de formes du subjonctif. Les auxiliares au subjonctif, à la différence de l'indicatif, se combinent, au moins dans les registres soutenus, non pas avec des participes (imperfectif : ikus-ten "voyant", perfectif : ikus-i "vu"), mais avec le radical du verbe lexical (ikus). E13 montre, à titre d'illustration, la séquence ikus dezan "qu'il (le) voie".

E13. Joan, bai, joan, eta argazki-a-k egin, mundu-a-k ikus dezan.

BAS aller oui aller et photo-DET-PLin faire monde-DET-ERG voir PRS.SUBJ 3.SG $<3 . S G$

"Allez-y, oui, allez-y, et faites des photos, pour que le monde le voie."

(Euskaldunon Egunkaria 11/01/02)

Pour construire les auxiliaires à valeur potentielle, les bases des formes du subjonctif se combinent avec le suffixe $-k e$. La relation structurelle entre les différentes paradigmes est illustrée par T3. 
T3. La relation structurelle entre subjonctif et potentiel dans les auxiliaires transitifs

\begin{tabular}{|llll|}
\hline personne & indicatif présent & $\begin{array}{c}\text { subjonctif présent } \\
\text { non-potentiel }\end{array}$ & $\begin{array}{c}\text { subjonctif } \\
\text { présent potentiel }\end{array}$ \\
\hline $1 . \mathrm{SG}$ & $d u-t$ & deza-da-n & deza-ke-t \\
\hline 2.SG & $d u-z u$ & deza-zu-n & deza-ke-zu \\
\hline 3.SG & $d u$ & deza-n & deza-ke \\
\hline $1 . \mathrm{PL}$ & $d u-g u$ & deza-gu-n & deza-ke-gu \\
\hline 2.PL & $d u-z u e$ & deza-zue-n & deza-ke-zue \\
\hline $3 . \mathrm{PL}$ & $d u-t e$ & deza-te-n & deza-ke-te \\
\hline
\end{tabular}

A l'aide de ces paradigmes, on peut obtenir l'opposition suivante :

ikusten duzu

ikus dezazun

ikus dezakezu "tu (le) vois"

"pour que tu (le) voies"

"tu peux (le) voir"

Notons en passant que les formes correspondantes du biscayen, le dialecte le plus occidental, sont assez différentes de celles des autres dialectes. T4 compare les paradigmes des auxiliaires transitifs du potentiel en guipuscoan et en biscayen. Celui-ci constitue un dialecte "périphérique" qui, de nos jours, est peu représenté à l'écrit où l'on préfère en général les formes "standardisées", plus proches du guipuscoan et du navarro-labourdin.

T4. Les formes du potentiel en guipuscoan et en biscayen

\begin{tabular}{|llc|}
\hline personne & guipuscoan & biscayen \\
\hline $1 . S G$ & dezaket & neinke \\
\hline $2 . \mathrm{SG}$ & dezakezu & zeinke \\
\hline $3 . \mathrm{SG}$ & dezake & leike \\
\hline $1 . \mathrm{PL}$ & dezakegu & geinke \\
\hline 2.PL & dezakezute & zeinkie \\
\hline
\end{tabular}




\subsection{Le potentiel de l'indicatif : du futur à l'épistémique}

En basque classique et dans certains parlers du nord, on trouve le suffixe - $k$ e en combinaison avec les radicaux de l'indicatif. Les formes correspondant à dezake et daiteke sont respectivement duke et dateke. Dans les textes classiques, elles servent à exprimer un futur duratif ou indéterminé (Lafon 1972/1999 : 515). Pour cette raison, ces constructions sont parfois appelées 'futur archaïque' (Zubiri \& Zubiri 2000 : 440).

E14. Dohatsu izan-en zarete nehor-k injuria

BAS heureux AUX.INTR-FUT PRS.2.PL quelqu'un-ERG injure

\begin{tabular}{|c|c|c|c|}
\hline erran & draukezue-n-ean, & persekuta-tu & zaitukezte-n-ean \\
\hline dire & PRSPOTCOI2PLRE_LOC et & persécuter-PART & PRS.POT-REL-LOC \\
\hline & $3 . \mathrm{SG}<3 . \mathrm{SG}$ & & 2.PL $<3 . \mathrm{SG}$ \\
\hline
\end{tabular}

eta hitz gaixto guzi-a erran dukeite-n-ean zuen kontra,

et mol mal tout-DET dir PRS.POT-REL-LOC POSS.2.PL contre

\section{SG<3.PL}

gezurr-ez

ene kausa-z.

mensonge-INSTR POSS.1.SG cause-INSTR

“Heureux serez-vous, lorsqu'on vous outragera, qu'on vous persécutera et qu' on dira faussement de vous toute sorte de mal, à cause de moi."

(Leizarraga 1571, Testamentu Berria)

E15. Baina Jaun-agana konberti-tu dateke-en-ean ken-du-ren da estalki-a.

BAS mais seigneur-ALL convertir-PART PRS.POT-REL-LOC ôter-PART-FUT PRS voile-DET

3.SG $3 . S G$

"Mais lorsqu'il (=le cour) se convertira au Seigneur, le voile sera ôté."

(Leizarraga 1571, Testamentu Berria)

En E14 et E15, les formes auxiliaires draukezue, zaitukezte, dukeite et dateke se combinent avec le participe du perfectif, mais elles peuvent se combiner également avec le suffixe aspectuel du prospectif/futur -(r)en (E16), ce qui donne un futur 'double’ (Lafon 1972/1999 : 517). 


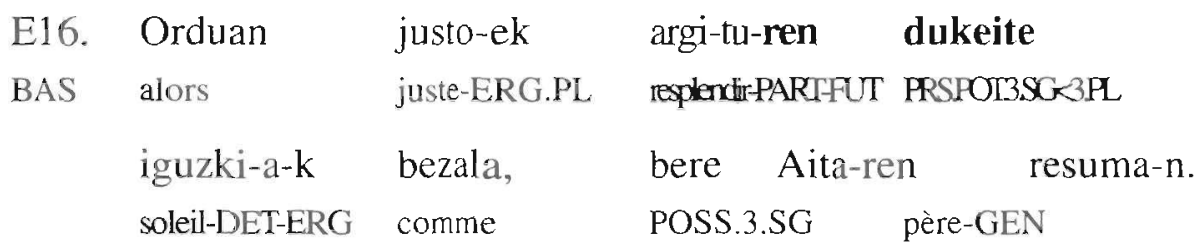
royaume-LOC

"Alors les justes resplendiront comme le soleil dans le royaume de leur Père."

\section{(Leizarraga 1571, Testamentu Berria)}

Trois ou quatre siècles plus tard, ces formes n'expriment plus le futur (sauf en souletin, le dialecte le plus oriental, cf. Rebuschi 1997: 140), mais transforment, en navarro-labourdin, une affirmation en hypothèse (comme dans E17 et E18). La marque de futurité est ainsi devenue une marque de modalitế épistémique.
E17. Ikerketa-ren
lehen
uste-etan
BAS recherche-GEN
premier
croyance-LOC.PL

gaizki hil zigarreta bat-ek du-ke su-a zabal-du.

mal tuer cigarette un-ERG PRS-POT feu-DET répandre-PART

\section{$3 . \mathrm{SG}<3 . \mathrm{SG}$}

"Selon les premières hypothèses de l'enquête, une cigarette mal éteinte aurait/pourrait avoir répandu le feu."

(www.herriak.info/lapurdi)

E18. Oraino ere orhoi-tze-n dateke.

BAS maintenant aussi souvenir-NR-LOC PRS.POT.3.SG

"Il s'en souvient probablement encore aujourd'hui."

(Lafitte 1944/2001: 369). 


\subsection{Le potentiel du subjonctif : de la capacité à l'épistémique}

En basque moderne, les formes du potentiel construites sur la base du subjonctif (dezakeldaiteke etc.) sont souvent employées dans des contextes épistémiques. E19 montre un tel cas.

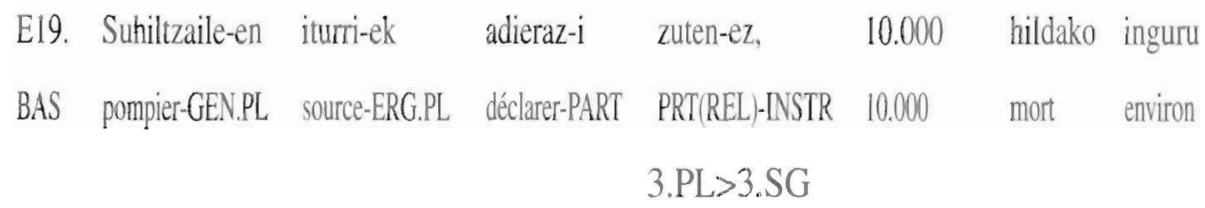

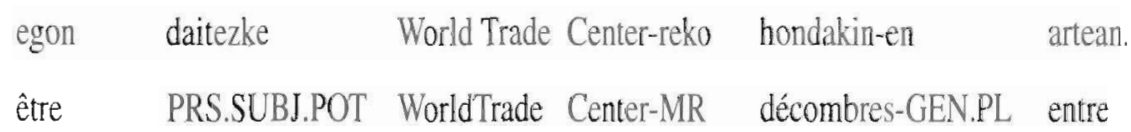

3.PL

"Selon les déclarations des pompiers, il se peut qu'environ 10.000 morts se trouvent sous les décombres du World Trade Center."

(Euskaldunon Egunkaria 13/09/01)

Il semble pourtant qu'à un stade plus ancien, ces formes étaient plus rarement employées pour exprimer une conjecture. Tant que le potentiel de l'indicatif ( $d u k e$ etc.) exprimait la modalité épistémique, le potentiel du subjonctif (dezake etc.) était essentiellement employé pour parler d'une capacité du référent du sujet. Mais comme les formes du type duke/dateke ont disparu du sud et sont devenues rares au nord, leur domaine fonctionnel est resté orphelin en quelque sorte. Une confusion entre les deux paradigmes en a été la conséquence. Au $18^{\mathrm{e}}$ siècle, des constructions avec le potentiel du subjonctif, comme par exemple eri ditake (=daiteke) "il peut tomber malade", commencent à remplacer le potentiel de l'indicatif, eri dateke "il est probablement malade" (Lafitte 1944 : 269). Au sud, on utilise en outre beaucoup le futur pour exprimer une probabilité plus forte.

On peut alors supposer qu'autrefois, dezake et daiteke étaient réservés, ou du moins privilégiés, pour l'expression d'un pouvoir physique, puisque les formes du type duke et dateke servaient à exprimer la conjecture (Lafitte 1944 : 369). Lorsque ces formes sont tombées en désuétude, il ne restait que les formes du potentiel du subjonctif pour exprimer une possibilité épistémique. 
Il semble que la particule ahal subit le même changement sémantique dans certaines variétés du nord où les constructions périphrastiques ont largement remplacé les auxiliaires complexes. Ainsi en E20, ahal a une interprétation épistémique puisqu'il s'agit d'une éventualité.

E20. Bainan gehi-en-ek diote egun hobe batzu-en

mais plus-EXCESS-ERG.PL direPRS.3.PL jour meilleur quelques-GEN.PL

$\begin{array}{lcclll}\text { mentura-n } & \text { geldi-tze-a } & \text { nagusi } & \text { de-la, } & \\ \text { chance-LOC rester-NR-DET } & \text { principal } & \text { PRS3.SG-SR } & & \\ \text { gazte } & \text { batzu-en } & \text { burraso-a-k } & \text { bereziki } & \text { etziren } & \text { batere bero } \\ \text { jeune } & \text { quelques-GEN } & \text { parents-DET-PL } & \text { particulièrement } & \text { NEG:PRT.3.PL du.tout chaud } \\ \text { beren } & \text { gazte-txo-en } & \text { Arjentina-rat } & \text { uz-te-ko, }\end{array}$

POSS.3.PL.REFL jeune-DIM-GEN.PL Argentine-ALL laisser-NR-MR

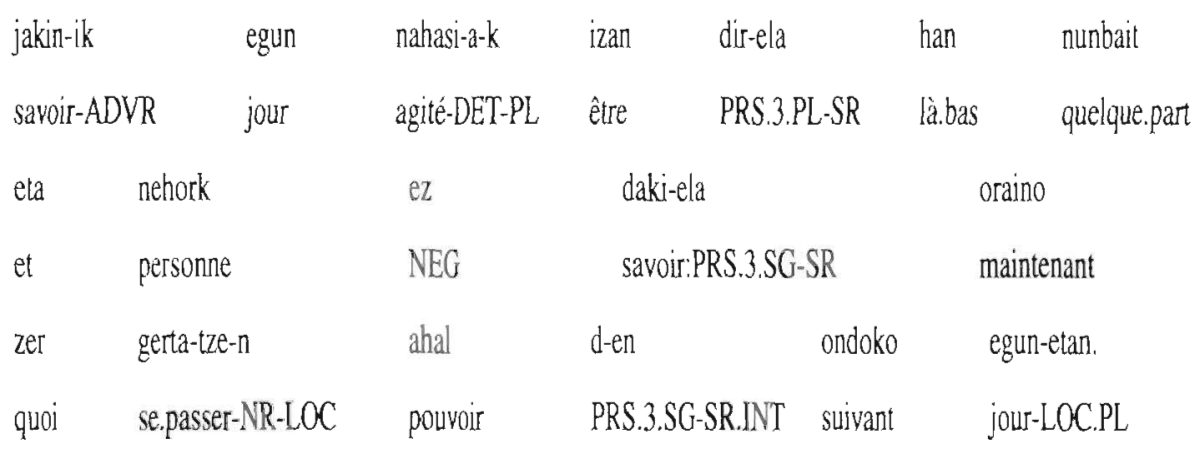

"Mais la plupart disent qu'il vaudrait mieux attendre un meilleur moment, particulièrement les parents de certains jeunes n'étaient pas du tout favorables à laisser leurs enfants aller en Argentine, sachant que la situation est tendue à certains endroits et que personne ne sait maintenant ce qui peut se passer dans les jours suivants." (www.herriak.info/lapurdi)

\subsection{La confusion entre subjonctif et indicatif dans les formes hypothétiques}

En basque, le verbe conjugué peut apparaître dans trois temps-modes: le présent, le passé, et l'hypothétique. Pour l'instant, on n'a vu que des formes au présent. A l'hypothétique, le suffixe -ke peut s'attacher soit aux formes de l'indicatif, soit à celles du subjonctif. T5 montre les formes correspondantes pour un sujet de $1^{\text {re }}$ personne singulier. 
T5. Les formes du potentiel au temps-mode hypothétique

\begin{tabular}{|lll|}
\hline & intransitif & transitif \\
\hline indicatif & nintzateke & nuke \\
\hline subjonctif & ninteke & nezake \\
\hline
\end{tabular}

L'emploi de ces formes est différent selon les régions. Au sud, les formes du subjonctif expriment la possibilité au conditionnel, comme ninteke dans E21.

E21. Bihar joan ninteke, nahi ba-duzu.

BAS demain aller HYP.POT.SUBJ vouloir COND- PRS

1.SG $\quad 3 . S G<2 . S G$

"Je pourrais aller demain, si tu veux." (King 1994 : 381)

Dans les dialectes du nord, ces formes remplacent le conditionnel simple (E22).

E22. Aberats-a (iza-te-n) ba-nintz, etxe handi bat eros nezake.

BAS riche-DET être-NR-LOC COND-HYP maison grand un acheter HYP.POTSUBJ

1.SG 1.SG $>3 . S G$

"Si j'étais riche, j’achèterais une grande maison." (King 1994 : 261)

Pour exprimer un tel conditionnel, les variétés du sud emploient les formes de l'hypothétique à l'indicatif qui se combinent avec le verbe lexical au futur (E23)

$\begin{array}{llllll}\text { E23. Bat } & \text { aurki-tu } & \text { ba-nu, } & \text { eros-i-ko } & \text { nuke. } \\ \text { BAS } & \text { un } & \text { trouver-PART } & \text { COND-HYP } & \text { acheter-PART-FUT } & \text { HYP.POT } \\ & & & 1 . S G>3 . S G & & 1 . S G>3 . S G\end{array}$

"Si j'en trouvais un, je l'achèterais." (King 1994 : 261)

T6 résume les différentes valeurs. Puisque les variétés du nord emploient les formes ninteke/nezake pour exprimer un conditionnel simple, elles ont recours à la particule ahal pour exprimer une possibilité au conditionnel. 
T6. Les formes du potentiel au temps-mode hypothétique

\begin{tabular}{|lll|}
\hline variété & conditionnel "simple" & conditionnel potentiel \\
\hline nord & eros nezake & erosten ahalko nuke \\
\hline sud & erosiko nuke & eros nezake \\
\hline français & "je l'achèterais" & "je pourrais l'acheter" \\
\hline
\end{tabular}

Dans les variétés du sud-ouest, le potentiel au présent et à l'hypothétique (dezake vs, nezake, daiteke vs. liteke, etc.) ne sont pas toujours bien distingués dans leurs emplois, et il peut y avoir confusion entre les deux (King $1993: 248$ ).

\subsection{Les verbes lexicaux synthétiques}

Un autre changement concerne la disparition de la conjugaison synthétique, autrement dit la conjugaison sans auxiliaires. Dans les textes anciens, on trouve ainsi le suffixe -ke directement attaché à des verbes lexicaux. Cela donne des formes comme dagoke (E24 et E25) au lieu de egon daiteke ou egoiten ahal da, et dabilke (E24) au lieu de ibil daiteke ou ibiltzen ahal da.

$\begin{array}{llll}\text { E24. Zahar-ra } & \text { ezin } & \text { barur } & \text { diteke, } \\ \text { BAS vieux-DET } & \text { NPOT } & \text { jeûne } & \text { PRS.SUBJ.POT } \\ & & & 3 . S G \\ \text { belhauriko } & \text { orazino-tan } & \text { ezin } & \text { dago-ke, } \\ \text { agenouillé } & \text { oraison-LOC.PL } & \text { NPOT } & \text { être:PRS.3.SG-POT } \\ \text { erromeria-n } & \text { ezin } & \text { dabil-ke. } & \\ \text { pèlerinage-LOC } & \text { NPOT } & \text { marcher:PRS-POT } \\ & & 3 . S G\end{array}$

"Le vieux ne peut pas faire de jeûne, il ne peut pas rester agenouillé pendant les oraisons, il ne peut pas aller en pèlerinage." (Axular 1643, Gero)

$\begin{array}{lllllll}\text { E25. } & \text { Gure } & \text { gogo-a } & \text { ezin } & \text { dago-ke } & \text { gogoeta gabe; } \\ \text { BAS } & \text { POSS.1.PL } & \text { esprit-DET } & \text { NPOT } & \begin{array}{l}\text { etre-POT } \\ \text { pensée sans }\end{array} \\ & & & & \text { PRS.3.SG } & & \end{array}$




$\begin{array}{lllll}\text { ezin } & \text { gaute-ke } & \text { zerbait-etan } & \text { pensa-tu } & \text { gabe. } \\ \text { NPOT } & \text { être-POT } & \text { quelque.chose-LOC } & \text { penser-PART } & \text { sans } \\ & \text { PRS.3.SG } & & & \end{array}$

"Notre esprit ne peut pas rester sans pensée ; nous ne pouvons pas rester sans penser à quelque chose." (Axular 1643, Gero)

Ces formes sont extrêmement rares dans la langue moderne. Certaines formes peuvent survivre dans des expressions figées, comme dans ez dagoke "il ne peut rester en place" (comm. pers. Xarles Videgain).

\section{Constructions sans $-k e$}

\subsection{Le verbe modal -iro(n)}

On peut encore mentionner qu'en plus des auxiliaires et des particules il existait un verbe modal iron "pouvoir" (cf. Lafitte 1944 : 312-313). Il s' agit d'un verbe transitif dont la forme de citation, 'iron, n'est qu'une reconstruction artificielle qui n'existe pas dans la langue. Ce verbe se combinait également avec ahal. Dans E26, on peut voir la forme de la $3^{e}$ personne du singulier au présent du verbe iron qui est diro et qui correspond à dezake.

$\begin{array}{llllll}\text { E26. Utz-i } & \text { ahal } & \text { diro } & \text { gizon-a-k } & \text { bere } & \text { emazte-a } \\ \text { BAS laisser-PART pouvoir } & \text { pouvoir:PRS.3.SG homme-DET-ERG } & \text { POSS3.REFL } & \text { femme-DET } \\ \text { ezein } \quad \text { ere } & \text { kausa-gatik? } & & \\ \text { quelconque aussi } & \text { cause-MTV } & & \end{array}$

"Est-il permis à un homme de répudier sa femme pour un motif quelconque?" (Leizarraga 1571, Testamentu Berria)

De nos jours, le verbe iron est assez rare en dehors de la Soule, la partie la plus orientale.

\subsection{La périphrase avec eduki "avoir"}

Dans certaines zones, on trouve encore une autre construction pour exprimer une possibilité. Il s'agit d'une périphrase constituée de l'infinitif nominalisé du verbe lexical, marqué par la terminaison -t(z)ea, et du verbe eduki "avoir" (cf. Zubiri\&Zubiri 2000 : 457). Au lieu de eduki, on y trouve aussi les formes du verbe ukan/izan.'

$\begin{array}{lllll}\text { E27. Beste alde bat-etik, bakoitz-a-k bere lagun-arte-ko hizkera-n } \\ \text { BAS autre côté un-ABL chacun-DET-ERG } & \text { POSS.3.REFL ami-entre-MR parler-LOC }\end{array}$ 


$\begin{array}{lllll}\text { nahi du-en bezala } & \text { hitz } & \text { egi-te-a } & \text { dauka } \\ \text { vouloir } & \text { PRS-REL comme } & \text { parole } & \text { faire-NR-DET } & \text { avoir } \\ & 3 . S G<3 . S G & & & 3 . S G<3 . S G\end{array}$

“ $D$ 'autre part, chacun a la possibilité de parler comme il veut dans son parler informel" (http://groups.yahoo.com/group/euskaraz/message/193)

Cette construction est particulièrement fréquente lorsque la possibilité est en dehors du contrôle du protagoniste (King 1993 : 274). Pour E27, cela signifie que le fait de "parler comme on veut" ne dépend pas des compétences du locuteur, mais que c'est l'environnement socio-linguistique qui le permet. Cette préférence d'usage a son origine dans la genèse de cette construction. En effet, la construction hitz egitea dauka en E27 est une contraction de hitz egiteko modua dauka, où l'on pourrait traduire modua, selon les cas, par "possibilité, occasion, manière de faire" etc.

\section{Résumé et conclusion}

L'expression des notions modales de possibilité, de capacité et de l'épistémique a considérablement évolué depuis le $16^{\mathrm{e}}$ siècle. Le nombre des différentes formes du potentiel en -ke s'est réduit. Certaines formes ont été fossilisées et ont adopté un sémantisme plus restreint. En basque ciassique, les particules étaient nécessaires pour désambiguer les diverses fonctions des auxiliaires au mode du potentiel. En basque moderne, on emploie soit la particule, soit l'auxiliaire complexe, mais rarement les deux ensemble. Les formes du type duke qui servaient à l'expression d'un futur indéterminé sont devenues des marqueurs épistémiques. Mais comme ces formes disparaissent aussi, les auxiliaires du type dezake sont souvent employés à leur place. Par contre, il reste difficile d'employer ahal dans un contexte épistémique (E30). Alors que les constructions avec l'auxiliaire et celles avec la particule sont équivalentes lorsque le contexte n'est pas épistémique (E28), des formes en ke sont préférées lorsqu'il ne s'agit pas d'une capacité, mais d'une hypothèse, comme dans E29.

E28. Erran nahi baita, idatzi-a Euskal Herri oso-a-n

BAS dire vouloir parce.que:PRS.3.SG écrit-DET Basque pays entier-DET-LOC

$\begin{array}{llllll}\text { erabil daiteke-en } & \text { hizkuntza } & \text { de-la, } & \text { eta } & \text { aldi-z, } \\ \text { employer } & \text { PRS.SUBJ.POT.3.SG-REL } & \text { langue } & \text { PRS.3.SG-SR et } & \text { fois-INSTR } \\ \begin{array}{l}\text { bakoitz-a-k } \\ \text { du-ela }\end{array} & \text { bere } & \text { toki-a-n } & \text { balia-tze-n } & \text { ahal }\end{array}$


chacun-DET-ERG POSS.3.REFL endroit-DET-LOC employer-NR-LOC pouvoir RS.3.SG<3.SG-SR

$\begin{array}{ll}\text { bere } & \text { euskalki-a } \\ \text { POSS.3.REFL } & \text { dialecte.basque-DET }\end{array}$

"Parce que cela veut dire que l'écrit est la langue qui peut être employée dans le Pays basque entier, et que, d'autre part, chacun peut employer son dialecte dans sa région." (Argia 16/09/01)

E29. Azerbaijan-dik Turkia-ko kostalde-ra doa-ke-en/joan daiteke-en BAS Azerbaïdjan-ABL Turquie-MR côte-ALL aller:PRS.3.SG-POT-REL 3.SG

olio-bide-a eraiki nahi luke, Errusia saihes-tu-z. huile-chemin-DET construire vouloir HYP.POT Russie éviter-PART-INSTR. 3.SG<3.SG

"Il voudrait construire l'oléoduc qui irait d'Aserbaïdjan en Turquie, en contournant la Russie."

E30. Azerbaijan-dik Turkia-ko kostalde-ra joa-te-n ahal de-n

BAS Azerbaïdjan-ABL Turquie-MR côte-ALL aller-NR-LOC pouvoir PRS.3.SG-REL olio-bide-a eraiki nahi luke, Errusia saihes-tu-z. huile-chemin-DET construire vouloir HYP.POT Russie éviter-PART-INSTR 3.SG<3.SG

"Il voudrait construire l'oléoduc qui peut aller d'Aserbaïdjan en Turquie, en contournant la Russie."

I Ukan est la forme de citation au nord, izan au sud. La distinction entre un "avoir' lexical (eduki) et grammatical (ukan/izan) est typique des variétés du sud où elle correspond à la distinction que fait le castillan entre tener et haber. Au nord, ukan a les deux sens, tandis que eduki y a un sémantisme plus restreint qu'en basque méridional (cf. la différence entre français tenir et castillan tener). 


\section{Abréviations}

\section{Abréviations dans les traductions interlinéaires}

$\begin{array}{llll}1 & \text { Locuteur } & \begin{array}{c}\text { INT } \\ \text { tion }\end{array} & \text { Marque d'interroga- } \\ 2 & \text { Allocutaire } & \text { INTR } & \text { Intransitif } \\ 3 & \text { Délocutif } & \text { LOC } & \text { Locatif } \\ \text { ABL } & \text { Ablatif } & \text { MR } & \text { Modifieur } \\ \text { ABS } & \text { Absolutif } & \text { MTV } & \text { Motivatif } \\ \text { ADVR } & \text { Morphème adverbialisa- } & \text { NÉG } & \text { Négation } \\ \text { teur } & & \text { NPOT } & \text { Nonpotentiel } \\ \text { AFF } & \text { Affirmatif } & \text { NR } & \text { Morphème nominalisa- } \\ \text { ALL } & \text { Allatif } & \text { teur } & \\ \text { AUX } & \text { Auxiliaire } & \text { PART } & \text { Participe } \\ \text { COI } & \text { Complément d'objet indi- } & \text { PL } & \text { Pluriel } \\ \text { rect } & & \text { POSS } & \text { Possessif } \\ \text { COND } & \text { Conditionnel } & \text { POT } & \text { Potentiel } \\ \text { D } & \text { Déictique } & \text { PRS } & \text { Présent } \\ \text { DÉT } & \text { Déterminé } & \text { PRTF } & \text { Partitif } \\ \text { DIM } & \text { Diminutif } & \text { REFL } & \text { Réflexif } \\ \text { ERG } & \text { Ergatif } & \text { REL } & \text { Relatif } \\ \text { EXCESS } & \text { Excessif } & \text { SG } & \text { Singulier } \\ \text { FUT } & \text { Futur } & \text { SR } & \text { Subordinateur } \\ \text { GÉN } & \text { Génitif } & \text { SUBJ } & \text { Subjonctif } \\ \text { HYP } & \text { Hypothétique } & & \\ \text { INSTR } & \text { Instrumental } & & \end{array}$




\section{Bibliographie}

Charritton Zabaltzagarai, Pierre \& Haritschelhar Duhalde, Jean (eds.) 1999, Vasconiana. Bilb(a)o : Euskaltzaindia (Iker, 11).

King, Alan R. 1993, Communicative grammar of the Basque verb. Thèse de doctorat, University of London.

King, Alan R. 1994, The Basque language. A practical introduction. Reno: University of Nevada Press.

Lafitte, Pierre 1944/2001, Grammaire basque (Navarro-Labourdin littéraire). Donostia/Baiona: Elkarlanean.

Lafon, René 1970/1999, "Le suffixe - $k e$, -te dans la conjugaison basque". BSL 65 : 184-212. Réédité dans : Charritton \& Haritschelhar (eds.) $1999 ; 479-504$.

Lafon, René 1972/1999, "Le suffixe -ke, -te dans la conjugaison basque (suite et fin)". BSL $67: 239-265$. Réédité dans : Charritton \& Haritscheíhar (eds.) 1999; 505-528.

Rebuschi, Georges 1997, «Énoncés et formes hypothétiques en basque contemporain». Essais de linguistique basque. Bilbao: Université du Pays Basque \& Saint-Sébastien: Diputación Foral de Gipuzkoa (Supplements of Anuario del Seminario de Filología Vasca «Julio de Urquijo», XXXV); 139-154.

Zubiri, Entzi \& Zubiri, Ilari 2000, Euskal Gramatika Osoa. [Grammaire complète du basque] Bilb(a)o: Didaktiker.

\section{Remerciements}

Une grande partie des exemples et des analyses du présent article ont été présentés au First Vasconian Meeting on Semantics and Pragmatics qui a eu lieu à Bayonne le 23 et le 24 mai 2003. Je remercie les participants à ces journées pour leurs remarques. Je tiens à remercier particulièrement Michel Aurnague, Andrée Borillo, Alan King et Xarles Videgain pour leurs conseils sur des versions antérieures de cet article. Je voudrais aussi exprimer ma gratitude à la fondation Gottlieb Daimler et Karl Benz pour avoir financé mes recherches sur le basque, et à l'Equipe de Recherche en Syntaxe et Sémantique à l’Université de Toulouse-Le Mirail pour son soutien. 